\section{Histochemistry of the Gram-staining Reaction for Micro-organisms}

IN 1884 a Dane, Christian Gram, while working in Berlin, discovered a method of staining micro-organisms which has gone under his name ever since that date $^{1}$. Although the method is in daily use by bacteriologists of various nationalities all over the world, and has by reason of its importance in ordinary routine diagnosis been the subject of extensive research, it may be fairly said that it has never been adequately explained.

The method consists in staining heat-fixed smears with an aqueous solution of a dye of the pararosaniline series, mordanting with aqueous iodine, washing with alcohol until no more of the blue dye can be extracted and then counterstaining with a red dye of the acid fuchsin series.

Organisms which after extraction retain the blue dye are described as being Gram-positive, while those which are decolorized by the alcoholic extraction and take up the red dye are said to be Gramnegative. Obviously there must be some marked chemical or physical differences in the two groups of micro-organisms in order to account for such diverse staining reactions. Special interest is attached to the underlying mechanisms since it has been shown ${ }^{2}$ that dramatic differences exist in the selective bacteriostatic and bactericidal action of some of the newer anti-bacterial agents. Thus, for example, basic dyes, cationic detergents ${ }^{3}$, penicillin, gramicidin, etc., are much more active against Gram-positive species, anionic detergents inhibit the metabolism of Gram-positive organisms only, while tellurites, azides, etc., appear to be selective against Gramnegative species.

As a result of investigations carried out conjointly, we have observed that Gram-positive organisms can be rendered Gram-negative by a variety of methods, for example, by the action of certain carbohydratesplitting enzymes and by extraction processes. We have devised a method whereby organisms, for example, $C l$. welchii, can be 'stripped' of an essential part of their Gram-positive material and separated into Gram-negative cytoskeletons and a Gramnegative extract. Furthermore, we have developed a simple technique whereby a fraction from the extract can be 'plated' back on to the cytoskeletons to reconstitute Gram-positive bacilli. We have been able to show that the essential constituent in Grampositive organisms which differentiates them from those which are Gram-negative lies in their having as part of their surface structure the magnesium salt of ribonucleic acid.

The method of extracting this material utilizes the action on washed cells at $60^{\circ}$ of a 2 per cent aqueous solution of a bile salt in presence of oxygen. Material in the extract could be precipitated by alcohol, and fractionation methods showed that it consisted essentially of magnesium ribonucleate together with inert polysaccharides and traces of proteins.

The fundamental requisite for the successful recombination of magnesium ribonucleate with the Gram-negative cytoskeleton material is that the latter must be in a reduced condition. A number of reducing agents have proved suitable for this preliminary reduction, but all our recent experiments have been carried out with 1 per cent formalin in 0.8 per cent sodium chloride, in which the cyto- skeletons can be kept in satisfactorily receptive condition for several days. With exposure to air the cytoskeletons become irreversibly oxidized and recombination does not occur. Of particular interest is our observation that it has not yet been found possible to combine Gram-negative organisms with salts of ribonucleic acid.

In further experiments, brewer's yeast, Saccharomyces cerevisice, was subjected to the same extraction method and it was found that the extraction was essentially complete in 2-3 hours. The normal yeast cell was coated with a layer of Gram-positive material which stained a deep blue-black, and it was only when this was removed that the cytoplasm and the large nucleus it contained could be demonstrated by the Gram-staining method. The stripping of the yeast cell by the bile salt occurred in stages; as the blue-black surface material disappeared there was left a nucleated cell with a stippled surface. On further extraction the surface stippling and the nucleus were progressively bereft of their Grampositive material so that eventually the whole cell became. Gram-negative. The extract contained polysaccharides and magnesium and sodium salts of ribonucleic acid.

Magnesium ribonucleate from the extract, or from extracts of other organisms, could successively be plated back on to the reduced yeast cytoskeletons so that their nuclei became once more Gram-positive and the surface stippling also was restored. If one replaced the magnesium ribonucleate by the sodium salt this did not readily combine with the surface of yeast cytoskeletons, the results with the sodium salt being irregular and chiefly confined to plating the nuclei after prolonged contact. In no case could plating be achieved with salts of desoxyribonucleic acid or with nucleosides or nucleotides.

In the course of our experiments it has been shown that certain Gram-positive organisms, for example, Streptococcus salivarius, may become Gram-negative when grown in media in which the magnesium content is reduced to a minimum or in media where the production of acid is sufficient to prevent the formation of magnesium ribonucleate. In some cases these Gram-negative individuals display striking evidence of nucleation, and on sub-culture give rise on an agar surface to colonies which are rough, whereas their Gram-positive relatives produce colonies which are smooth.

The bearing of these facts on antigenic structure and relationships is under investigation, as also is the structure of the various cytoskeletons. Those from $C l$. welchii and from Saccharomyces cerevisice give a vivid Sakaguchi reaction and appear to contain a high proportion of basic protein material. We have, therefore, some evidence for the tentative suggestion that the Gram-positive (that is, dye-retaining) material is a high-molecular complex formed by the combination of a reduced basic protein substrate with magnesium ribonucleate.

City of Birmingham

H. HeNRy.

Bacteriological Laboratories.

Department of Chemistry,

University of Birmingham. May 12.

1 Gram, Fortschr. Med. (I), 2, S. 185 (1884).

Dubos, J. Bact.; 41, 269 (1941); Biochem. Rev., 659 (1942).

- Baker, Harrison and Miller, J. Exp. Med., 74, 611 (1941). 